\title{
FATORES DE RISCO PARA SEPSE NEONATAL EM UNIDADE DE TERAPIA: ESTUDO DE EVIDÊNCIA
}

\author{
Cecília Olívia Paraguai de Oliveira ${ }^{1}$, Juliana Raquel Silva Souza ${ }^{1}$, Regimar Carla Machado ${ }^{2}$ Alexsandra \\ Rodrigues Feijão ${ }^{2}$, Nilba Lima de Souza ${ }^{2}$
}

RESUMO: Revisão integrativa da literatura com o objetivo de apresentar as principais evidências de fatores de risco para sepse neonatal em recém-nascidos internados em Unidade de Terapia Neonatal. A busca foi realizada entre os meses de julho e agosto de 2015, nas bases de dados Scientific Eletronic Library Online, SciVerse Scopus e PubMed, utilizando como descritores: fatores de risco, unidade de terapia intensiva neonatal, recém-nascido e as seguintes palavras-chaves: sepse neonatal e prematuridade. A busca totalizou 31 publicações cujos resultados mostraram que os fatores preditivos para a sepse neonatal estão associados à idade gestacional, ruptura prematura das membranas amnióticas e infecção materna. Condições de nascimento, baixo peso e prematuridade são fortes evidências para sepse. Os fatores relacionados ao ambiente da unidade de terapia intensiva neonatal contribuem fortemente para a sepse tardia.

DESCRITORES: Enfermagem neonatal; Fatores de risco; Sepse; Unidades de Terapia Intensiva Neonatal.

\section{RISK FACTORS FOR NEONATAL SEPSIS IN NEONATAL INTENSIVE CARE UNIT: EVIDENCE STUDY}

ABSTRACT: Integrative literature review aimed to present the main evidence of risk factors for neonatal sepsis in newborns admitted to a Neonatal Intensive Care Unit. The search was performed between the months of July and August 2015, in the databases Scientific Electronic Library Online, SciVerse Scopus and PubMed, using the following descriptors: risk factors, neonatal intensive care unit, newborn and the following keywords: neonatal sepsis and prematurity. The search included 31 publications whose outcomes showed that the predictive factors for neonatal sepsis are associated to gestational age, premature rupture of amniotic membranes and maternal infection. Birth conditions, low weight and prematurity are strong evidence of sepsis. The factors related to the environment of the neonatal intensive care unit are potential contributing factors associated to late-onset sepsis.

DESCRIPTORS: Neonatal nursing; Risk factors; Sepsis; Neonatal Intensive Care Units.

\section{FACTORES DE RIESGO PARA SEPSIS NEONATAL EN UNIDAD DE TERAPIA: ESTUDIO DE EVIDENCIA}

RESUMEN: Revisión integrativa de literatura cuya finalidad fue presentar las principales evidencias de factores de riesgo para sepsis neonatal en recién nacidos internados en Unidad de Terapia Neonatal. La búsqueda fue hecha entre los meses de julio y agosto de 2015, en las bases de datos Scientific Eletronic Library Online, SciVerse Scopus y PubMed, utilizándose los descriptores: factores de riesgo, unidad de terapia intensiva neonatal, recién nacido; y las siguientes palabras claves: sepsis neonatal y prematuridad. La búsqueda totalizó 31 publicaciones cuyos resultados mostraron que los factores predictivos para sepsis neonatal están asociados a edad gestacional, ruptura prematura de las membranas amnióticas e infección materna. Condiciones de nacimiento, bajo peso y prematuridad son fuertes evidencias para sepsis. Los factores referentes al ambiente de la unidad de terapia intensiva neonatal contribuyen mucho para la sepsis tardía.

DESCRIPTORES: Enfermería neonatal; Factores de riesgo; Sepsis; Unidades de Terapia Intensiva Neonatal.

${ }^{1}$ Enfermeira. Mestra em Enfermagem. Universidade Federal do Rio Grande do Norte. Natal, RN, Brasil.

${ }^{2}$ Enfermeira. Doutora em Enfermagem. Docente de Enfermagem da Universidade Federal do Rio Grande do Norte. Natal, RN, Brasil. 


\section{INTRODUÇÃO}

A sepse neonatal é uma das principais causas de morte dos recém-nascidos ( $R N$ ) em todo mundo e configura-se como um dos fatores que mais contribui para a elevação do índice de mortalidade neonatal.

A incidência da sepse neonatal atinge cinco milhões de óbitos em $\mathrm{RN}$, sendo em sua maioria diagnosticada em países subdesenvolvidos ou em desenvolvimento, como o Brasil. Os sujeitos mais acometidos são os RN de baixo peso submetidos a procedimentos invasivos durante o período de permanência na Unidade de Terapia Intensiva Neonatal (UTIN) ${ }^{(1)}$.

A infecção pode ocorrer de forma precoce, manifestando-se até às 48 horas de vida, e após esse período caracteriza um acometimento tardio, usualmente causado por contato com patógenos adquiridos após o nascimento ${ }^{(2)}$.

Os sinais e sintomas são inespecíficos, de início silencioso, confundindo-se a condições próprias da idade, como alterações respiratórias, decorrentes da prematuridade. Observa-se ainda que, para cada recém-nascido verdadeiramente séptico, um grande número deles é tratado apenas por suspeita clínica presumida e não confirmada de infecção(1).

No Brasil, percebe-se ainda ausência de dados nacionais consolidados sobre a incidência de infecções em unidades neonatais incluindo a sepse. Há somente dados de estudos regionais, o que pode variar muito conforme o tipo de estabelecimento analisado. Os estudos nacionais publicados mostram que essas infecções estão relacionadas com o peso ao nascimento, a utilização de cateter venoso central (CVC) e com o tempo de ventilação mecânica ${ }^{(2)}$.

Nesse sentido, a investigação dos fatores de risco para a sepse neonatal é importante na implementação de medidas que qualifiquem a assistência prestada ao RN, e principalmente, a prevenção de riscos relativos à exposição a agentes patogênicos causadores da $\operatorname{sepse}^{(3)}$.

A realização do estudo fundamenta-se na necessidade de apropriar-se dos fatores de risco para a sepse neonatal, relevante para o direcionamento das ações de cuidado de enfermagem aos RN prematuros para redução da mortalidade nessa faixa etária.

O objetivo do estudo consiste em buscar as principais evidências disponíveis na literatura sobre os fatores de risco para a sepse neonatal em recém-nascidos internados em Unidades de Terapia Intensiva Neonatal.

\section{- MÉTODO}

Trata-se de uma revisão integrativa da literatura elaborada partindo de sua estrutura clássica, seguindo seis etapas: identificação do tema e formulação da questão norteadora, busca na literatura e seleção criteriosa das pesquisas, categorização dos estudos encontrados, análise dos estudos incluídos, interpretação dos resultados e comparações com outras pesquisas, e relato da revisão e síntese do conhecimento.

A questão norteadora proposta para esta revisão foi: "Quais os principais fatores de risco para sepse em recém-nascidos internados em UTIN?".

A etapa seguinte ocorreu com o levantamento bibliográfico de artigos científicos publicados e indexados nas seguintes bases de dados eletrônicas: Scientific Eletronic Library Online (SciELO), SciVerse Scopus (Scopus) e PubMed, utilizando como descritores: fatores de risco, unidade de terapia intensiva neonatal, recém-nascido; e as seguintes palavras-chaves: sepse neonatal e prematuridade. A opção por esses descritores e palavras-chaves deve-se ao fato de favorecer a busca de publicações referentes às particularidades do objeto de estudo.

Para definir a amostra foram utilizados os seguintes critérios de inclusão: estudos que abordavam os fatores de risco para sepse neonatal; artigos científicos originais indexados nas bases de dados selecionadas para o estudo, publicados nos idiomas português, inglês ou espanhol, disponíveis na 
íntegra com texto completo, e que utilizaram como descritores ou palavras-chave os já elencadas acima, no período de 2005 a 2015. Foram critérios de exclusão: artigos no formato de editoriais, cartas ao editor, opinião de especialistas, e aqueles que não se relacionavam com o objeto do estudo.

Foram estabelecidos e realizados os seguintes cruzamentos de modo a atender ao objetivo do estudo: Fatores de risco (AND) Sepse neonatal, Fatores de risco (OR) Sepse neonatal, Fatores de risco (AND) Unidade de Terapia intensiva neonatal, Fatores de risco (OR) Unidade de Terapia Intensiva Neonatal, Sepse neonatal (AND) Unidade de Terapia Intensiva neonatal, Sepse neonatal (OR) Unidade de Terapia Intensiva Neonatal, Sepse neonatal (AND) Recém-nascido, Sepse neonatal (OR) Recémnascido, Sepse neonatal (AND) Prematuridade, Sepse neonatal (OR) Prematuridade.

Em virtude das características específicas para o acesso de cada base de dados, as estratégias utilizadas para localizar os artigos foram adaptadas para cada uma, tendo como eixo norteador a pergunta e os critérios de inclusão, previamente estabelecidos, para manter a coerência na busca dos artigos e evitar possíveis vieses.

A busca foi realizada pelo acesso online, nos meses de julho e agosto de 2015, e utilizou-se um instrumento adaptado, com os seguintes indicadores: identificação do artigo original, características metodológicas do estudo, avaliação do rigor metodológico, das intervenções mensuradas e dos resultados encontrados.

Para análise dos artigos selecionados e apresentação dos resultados, elaboramos um quadro que contempla o título do artigo, autoria, delineamento do estudo, objetivo e principais resultados.

\section{RESUlTADOS}

A busca inicial gerou uma cifra de 2.597 artigos, que após aplicados os critérios de inclusão e exclusão totalizaram 107 publicações potenciais. Estas foram submetidas à leitura criteriosa para refinamento. Após análise dos artigos, foram selecionados 27 pertinentes a este estudo, e 80 foram excluídos por não atenderem aos critérios de inclusão. Aqueles encontrados repetidos em mais de uma base de dados foram considerados apenas uma vez.

Em sua maioria as publicações pertenciam a revistas internacionais, de pesquisas realizadas em unidades de terapia intensiva neonatais com o objetivo de investigar os fatores preditivos para o desfecho da sepse neonatal precoce ou tardia.

Quanto ao delineamento dos estudos avaliados, evidenciou-se na amostra: sete estudos seccionais descritivos $(22,6 \%)$, cinco estudos caso-controle (16,1\%), cinco estudos longitudinais observacionais $(16,1 \%)$, quatro estudos quantitativos retrospectivos $(12,9 \%)$, quatro revisões integrativas (12,9\%), três estudos de coorte $(9,7 \%)$, duas revisões narrativas $(6,5 \%)$ e uma revisão sistemática com metanálise (3,2\%). Os Quadros (1-4) a seguir expõem as características dos estudos analisados.

Quadro 1 - Estudos que relacionam sepse neonatal a fatores de risco materno. Natal, RN, Brasil, 2015 (continua)

\begin{tabular}{|c|c|c|c|}
\hline Autor & Objeto de estudo & Método & Resultado/Fator de risco \\
\hline $\begin{array}{l}\text { Berardi A, Cattelani } \\
\text { C, Creti R, Berner } \\
\text { R, Pietrangiolillo Z, } \\
\text { Immaculada M, et al. } \\
2015^{(1)}\end{array}$ & $\begin{array}{c}\text { Determinar se a profilaxia } \\
\text { antimicrobiana materna para } \\
\text { estreptococo do grupo B, até } \\
\text { quatro horas antes do parto, } \\
\text { aumenta o diagnóstico de } \\
\text { sepse neonatal clínica. }\end{array}$ & $\begin{array}{l}\text { Estudo quantitativo } \\
\text { retrospectivo }\end{array}$ & $\begin{array}{c}\text { Antibiótico profilaxia } \\
\text { materna até quatro horas } \\
\text { antes do parto reduz o risco } \\
\text { para sepse neonatal }\end{array}$ \\
\hline $\begin{array}{l}\text { Pinheiro RS, Ferreira } \\
\text { LCL, Brum IR, } \\
\text { Guilherme JP, Monte } \\
\text { RL, 2007(4) }\end{array}$ & $\begin{array}{l}\text { Identificar os fatores de } \\
\text { risco maternos envolvidos } \\
\text { na sepse neonatal precoce }\end{array}$ & $\begin{array}{l}\text { Estudo de coorte } \\
\text { longitudinal } \\
\text { prospectivo }\end{array}$ & $\begin{array}{l}\text { Ausência de seguimento } \\
\text { pré-natal, rompimento } \\
\text { prolongado da membrana e } \\
\text { infecção bacteriana }\end{array}$ \\
\hline $\begin{array}{c}\text { Goulart AP, Valle CF, } \\
\text { Dal-Pizzol F, Cancelier } \\
\text { ACL, 2006 }\end{array}$ & $\begin{array}{c}\text { Identificar os fatores de risco } \\
\text { associados à sepse neonatal } \\
\text { precoce }\end{array}$ & Estudo caso-controle & $\begin{array}{l}\text { Infecção materna, filho } \\
\text { anterior com sepse neonatal } \\
\text { e rompimento prolongado da } \\
\text { membrana }\end{array}$ \\
\hline
\end{tabular}


Quadro 1 - Estudos que relacionam sepse neonatal a fatores de risco materno. Natal, RN, Brasil, 2015 (continuação)

\begin{tabular}{|c|c|c|c|}
\hline Autor & Objeto de estudo & Método & Resultado/Fator de risco \\
\hline $\begin{array}{c}\text { Puopolo KM, Draper } \\
\text { D, Wi S, Newman TB, } \\
\text { Zupancic J, Lieberman } \\
\text { E, et al. } 2011^{(6)}\end{array}$ & $\begin{array}{l}\text { Estimar Sepse precoce de } \\
\text { acordo com fatores de risco } \\
\text { intrauterino }\end{array}$ & $\begin{array}{l}\text { Estudo de caso- } \\
\text { controle }\end{array}$ & $\begin{array}{c}\text { Aumento da temperatura } \\
\text { intrauterina }\end{array}$ \\
\hline $\begin{array}{l}\text { Alam MM, Saleem } \\
\text { AF, Shaikh AS, Munir } \\
\text { O, Qadir M, 2014 }\end{array}$ & $\begin{array}{c}\text { Relatar a incidência e } \\
\text { fatores de risco para a sepse } \\
\text { neonatal }\end{array}$ & $\begin{array}{c}\text { Estudo quantitativo } \\
\text { descritivo }\end{array}$ & $\begin{array}{l}\text { Infecção materna e ruptura } \\
\text { prolongada da placenta }\end{array}$ \\
\hline $\begin{array}{c}\text { Mukhopadhyay } \\
\text { S, Puopolo KM, 2012 }\end{array}$ & $\begin{array}{c}\text { Avaliar sinais clínicos e } \\
\text { fatores de risco para sepse } \\
\text { neonatal }\end{array}$ & $\begin{array}{l}\text { Estudo de Revisão } \\
\text { integrativa }\end{array}$ & $\begin{array}{c}\text { Idade gestacional }>37 \\
\text { semanas, ruptura prematura } \\
\text { da placenta e baixo peso ao } \\
\text { nascer são fatores de risco } \\
\text { para sepse neonatal }\end{array}$ \\
\hline $\begin{array}{c}\text { Chan GJ, Lee ACC, } \\
\text { Baqui AH, Tan J, Black } \\
\text { RE, 2013 } \\
\end{array}$ & $\begin{array}{l}\text { Identificar a transmissão } \\
\text { vertical da sepse neonatal }\end{array}$ & $\begin{array}{l}\text { Revisão sistemática } \\
\text { com metanálise }\end{array}$ & $\begin{array}{c}\text { Colonização materna e } \\
\text { ruptura prematura da } \\
\text { placenta }\end{array}$ \\
\hline Jiang Z, Ye Y, 2013 ${ }^{(24)}$ & $\begin{array}{c}\text { Investigar os principais } \\
\text { fatores de risco para sepse } \\
\text { precoce e traçar medidas de } \\
\text { prevenção }\end{array}$ & $\begin{array}{l}\text { Estudo de caso- } \\
\quad \text { controle }\end{array}$ & $\begin{array}{l}\text { Líquido amniótico e ruptura } \\
\text { prolongada da placenta }\end{array}$ \\
\hline
\end{tabular}

Quadro 2 - Estudos que relacionam sepse neonatal a fatores de risco do recém-nascido. Natal, RN, Brasil, 2015

\begin{tabular}{|c|c|c|c|}
\hline Autor & Objeto de estudo & Método & Resultado/Fatores de risco \\
\hline $\begin{array}{c}\text { Yilmaz NO, Agus } \\
\text { N, Helvaci M, Kose } \\
\text { S, Ozer E, Sahbudak } \\
\text { Z, 2010 }\end{array}$ & $\begin{array}{l}\text { Associar fatores de risco } \\
\text { com a mortalidade por sepse } \\
\text { neonatal }\end{array}$ & $\begin{array}{l}\text { Estudo quantitativo } \\
\text { observacional }\end{array}$ & $\begin{array}{c}\text { O peso ao nascer e infecção } \\
\text { por Klebsiella associam-se } \\
\text { à mortalidade por sepse } \\
\text { neonatal }\end{array}$ \\
\hline $\begin{array}{c}\text { Carvalho JK, Moore } \\
\text { DB, Luz RA, Xavier- } \\
\text { Elsas PP, Gaspar-Elsas } \\
\text { MIC, } 2013^{(13)}\end{array}$ & $\begin{array}{l}\text { Utilização da genotipagem } \\
\text { para identificar o risco de } \\
\text { sepse }\end{array}$ & Revisão narrativa & $\begin{array}{c}\text { Não foi identificada associação } \\
\text { positiva entre polimorfismos } \\
\text { específicos e risco aumentado } \\
\text { de sepse }\end{array}$ \\
\hline $\begin{array}{c}\text { Karambin } M \text {, Zarkesh } \\
M, 2010^{(14)}\end{array}$ & $\begin{array}{c}\text { Determinar a prevalência dos } \\
\text { agentes infecciosos da sepse } \\
\text { neonatal }\end{array}$ & Estudo prospectivo & $\begin{array}{l}\text { A prematuridade e o baixo } \\
\text { peso ao nascer associam-se à } \\
\text { sepse neonatal; }\end{array}$ \\
\hline $\begin{array}{c}\text { Seale AC, Mwaniki } \\
\text { M, Newton } \\
\text { CR, Berkley JA, } \\
2009^{(15)}\end{array}$ & $\begin{array}{c}\text { Identificar etiologia, } \\
\text { incidência e fatores de risco } \\
\text { para a sepse neonatal e } \\
\text { maternal na África. }\end{array}$ & $\begin{array}{l}\text { Estudo de revisão } \\
\text { narrativa }\end{array}$ & $\begin{array}{l}\text { Prematuridade e baixo peso } \\
\text { ao nascer são fatores de risco } \\
\text { para sepse }\end{array}$ \\
\hline $\begin{array}{c}\text { Gessner } \\
\text { BD, Castrodale } \\
\text { L, Soriano-Gabarro } \\
\text { M, 2005 }\end{array}$ & $\begin{array}{c}\text { Identificar etiologia da sepse } \\
\text { neonatal no Alasca }\end{array}$ & $\begin{array}{l}\text { Estudo de revisão } \\
\text { integrativa }\end{array}$ & $\begin{array}{l}\text { Prematuro com pneumonia } \\
\text { e baixo peso ao nascer estão } \\
\text { predispostos à infecção }\end{array}$ \\
\hline $\begin{array}{c}\text { Madan JC, Salari } \\
\text { RC, Saxena } \\
\text { D, Davidson } \\
\text { L, O'Toole GA, Moore } \\
\text { JH, et al. } 2011^{(26)}\end{array}$ & $\begin{array}{l}\text { Compreender a colonização } \\
\text { microbiana do prematuro. }\end{array}$ & $\begin{array}{l}\text { Estudo prospectivo } \\
\text { longitudinal }\end{array}$ & $\begin{array}{l}\text { Presença de mecônio em } \\
\text { recém-nascidos de baixo peso } \\
\text { produz risco aumentado para } \\
\text { sepse neonatal. }\end{array}$ \\
\hline
\end{tabular}


Quadro 3 - Estudos que relacionam sepse neonatal a fatores externos. Natal, RN, Brasil, 2015

\begin{tabular}{|c|c|c|c|}
\hline Autor & Objeto de estudo & Método & Resultado/Fatores de risco \\
\hline $\begin{array}{c}\text { Verstraete EH, De Coen } \\
\text { K, Vogelaers D, Blot S, } \\
2015^{(3)}\end{array}$ & $\begin{array}{l}\text { Descrever os fatores de } \\
\text { risco para infecção da } \\
\text { corrente sanguínea }\end{array}$ & Estudo de coorte & $\begin{array}{c}\text { Peso, nutrição parenteral } \\
\text { e ventilação mecânica são } \\
\text { fatores de risco }\end{array}$ \\
\hline $\begin{array}{c}\text { Romanelli RMC, Anchieta } \\
\text { LM, Carvalho EAA, Glória e } \\
\text { Silva LF, Nunes RVP, Mourão } \\
\text { PH, et al. } 2014^{(18)}\end{array}$ & $\begin{array}{c}\text { Identificar os fatores de } \\
\text { risco para sepse após } \\
\text { cirurgia }\end{array}$ & $\begin{array}{l}\text { Estudo de caso- } \\
\text { controle }\end{array}$ & $\begin{array}{c}\text { Ventilação mecânica, } \\
\text { prematuro submetidos à } \\
\text { cirurgia e duração da nutrição } \\
\text { parenteral }\end{array}$ \\
\hline $\begin{array}{l}\text { Li Z, Xiao Z, Li Z, Zhong } \\
\text { Q, Zhang Y, Xu F, 2013 }\end{array}$ & $\begin{array}{c}\text { Explorar os fatores de risco, } \\
\text { antibióticos para sepse } \\
\text { neonatal }\end{array}$ & $\begin{array}{l}\text { Estudo } \\
\text { quantitativo } \\
\text { retrospectivo }\end{array}$ & $\begin{array}{l}\text { O cateter central de inserção } \\
\text { periférica (PICC) foi o } \\
\text { principal fator de risco para a } \\
\text { sepse neonatal }\end{array}$ \\
\hline $\begin{array}{c}\text { Marchant EA, Boyce } \\
\text { GK, Sadarangani M, Lavoie } \\
\text { PM, 2013 }\end{array}$ & $\begin{array}{l}\text { Revisar a epidemiologia } \\
\text { dos fatores de risco } \\
\text { imunológicos da sepse } \\
\text { neonatal. }\end{array}$ & $\begin{array}{l}\text { Revisão } \\
\text { integrativa }\end{array}$ & $\begin{array}{l}\text { Procedimentos invasivos na } \\
\text { UTI são os principais fatores } \\
\text { de risco para a sepse neonatal }\end{array}$ \\
\hline $\begin{array}{l}\text { Turrentine MA, Greisinger } \\
\text { AJ, Brown KS, Wehmanen } \\
\text { OA, Mouzoon ME, } 2013^{(10)}\end{array}$ & $\begin{array}{l}\text { Identificar a associação do } \\
\text { uso empírico de antibiótico } \\
\text { com a sepse tardia }\end{array}$ & Estudo de coorte & $\begin{array}{c}\text { O uso empírico do antibiótico } \\
\text { relaciona-se com a piora do } \\
\text { quadro clínico }\end{array}$ \\
\hline $\begin{array}{c}\text { Lin CB, Hornik CP, Clark R, } \\
\text { Cotten CM, Benjamin Jr DK, } \\
\text { Cohen-Wolkoweiz M, et al , } \\
2012^{(23)}\end{array}$ & $\begin{array}{l}\text { Avaliar associação entre } \\
\text { cultura positiva e sepse } \\
\text { neonatal tardia }\end{array}$ & $\begin{array}{l}\text { Estudo } \\
\text { quantitativo } \\
\text { retrospectivo }\end{array}$ & $\begin{array}{c}\text { Nesse estudo não há } \\
\text { associação entre cultura } \\
\text { positiva para sepse neonatal e } \\
\text { início tardio da sepse }\end{array}$ \\
\hline $\begin{array}{c}\text { Leal YA, Álvarez-Nemegyei } \\
\text { J, Velázquez JR, Rosado- } \\
\text { Quiab U, Diego-Rodríguez } \\
\text { N, Paz-Baeza E, et al, } \\
2012^{(7)}\end{array}$ & $\begin{array}{l}\text { Identificar fatores de risco } \\
\text { para prognóstico da sepse } \\
\text { neonatal }\end{array}$ & $\begin{array}{l}\text { Estudo } \\
\text { quantitativo } \\
\text { descritivo }\end{array}$ & $\begin{array}{c}\text { Complicações respiratórias, } \\
\text { exigência de ventilação } \\
\text { assistida, e procedimento } \\
\text { cirúrgico são fatores de risco } \\
\text { para sepse neonatal }\end{array}$ \\
\hline $\begin{array}{l}\text { Hsieh E, Smith PB, Jacqz- } \\
\text { Aigrain A, Kaguelidou F, } \\
\text { Cohen-Wolkowiez M, } \\
\text { Manzoni P, et al. 2012(21) }\end{array}$ & $\begin{array}{l}\text { Identificar os fatores de } \\
\text { risco para sepse neonatal } \\
\text { relacionada à Candida e } \\
\text { relatar seu tratamento. }\end{array}$ & $\begin{array}{l}\text { Estudo } \\
\text { quantitativo } \\
\text { descritivo }\end{array}$ & $\begin{array}{l}\text { Cândida, exposição prévia às } \\
\text { cefalosporinas e presença de } \\
\text { cateteres venosos centrais são } \\
\text { fatores de risco para sepse. }\end{array}$ \\
\hline
\end{tabular}

Quadro 4 - Estudos que relacionam sepse neonatal a fatores externos. Natal, RN, Brasil, 2015

\begin{tabular}{|c|c|c|c|}
\hline Autor & Objeto de estudo & Método & Resultado/Fator de risco \\
\hline $\begin{array}{c}\text { Puopolo KM, Draper } \\
\text { D, Wi S, Newman TB, } \\
\text { Zupancic J, Lieberman } \\
\text { E, et al. } 2011^{(6)}\end{array}$ & $\begin{array}{l}\text { Estimar Sepse precoce de } \\
\text { acordo com fatores de risco } \\
\text { intrauterino }\end{array}$ & $\begin{array}{l}\text { Estudo de caso- } \\
\text { controle }\end{array}$ & $\begin{array}{c}\text { Correlação com sepse } \\
\text { neonatal: aumento da } \\
\text { temperatura intrauterina } \\
\text { e tempo de exposição ao } \\
\text { rompimento da placenta. }\end{array}$ \\
\hline $\begin{array}{c}\text { Goulart AP, Valle CF, } \\
\text { Dal-Pizzol F, Cancelier } \\
\text { ACL, 2006 }\end{array}$ & $\begin{array}{l}\text { Investigar os fatores de risco } \\
\text { ligado a sepsemia neonatal }\end{array}$ & $\begin{array}{l}\text { Estudo quantitativo } \\
\text { descritivo }\end{array}$ & $\begin{array}{l}\text { Alimentação enteral é fator } \\
\text { de risco para sepse }\end{array}$ \\
\hline $\begin{array}{c}\text { Meireles LA, Vieira } \\
\text { AA, Costa CR , 2010 }\end{array}$ & $\begin{array}{c}\text { Descrever características } \\
\text { clinicas de recém-nascido } \\
\text { com sepse tardia comprovada } \\
\text { e não comprovada }\end{array}$ & $\begin{array}{l}\text { Estudo quantitativo } \\
\text { descritivo }\end{array}$ & $\begin{array}{c}\text { Tempo total de internação e } \\
\text { presença de neutrófilos são } \\
\text { fatores de risco para a sepse } \\
\text { neonatal. }\end{array}$ \\
\hline $\begin{array}{c}\text { Borges RM, Soares LR, } \\
\text { de Brito CS, Brito DVD, } \\
\text { Abdallah VOS, Gontijo } \\
\text { Filho PP, 2009(25) }\end{array}$ & $\begin{array}{c}\text { Investigar a Candida albicans } \\
\text { como agente de colonização } \\
\text { e sepse, e os fatores de risco } \\
\text { para a sepse neonatal }\end{array}$ & $\begin{array}{l}\text { Estudo quantitativo } \\
\text { observacional com } \\
\text { dados secundários }\end{array}$ & $\begin{array}{l}\text { O uso prévio de antibiótico } \\
\text { e o cateter vascular central } \\
\text { umbilical são fatores de } \\
\text { risco para sepse neonatal }\end{array}$ \\
\hline $\begin{array}{l}\text { Tragante CR, Ceccon } \\
\text { MEJR, Falcão MC, Seiti } \\
\text { M, Sakita N, Vieira RA, } \\
2008^{(20)}\end{array}$ & $\begin{array}{c}\text { Determinar a prevalência } \\
\text { e a mortalidade de sepse } \\
\text { neonatal por bactérias Gram } \\
\text { negativas }\end{array}$ & $\begin{array}{l}\text { Estudo retrospectivo } \\
\text { e descritivo }\end{array}$ & $\begin{array}{l}\text { A mortalidade relacionada a } \\
\text { sepse foi maior por bactérias } \\
\text { gram negativas provenientes } \\
\text { do ambiente hospitalar. }\end{array}$ \\
\hline
\end{tabular}


Os resultados presentes nestas publicações mostraram que os fatores de risco para o desenvolvimento da sepse neonatal estão relacionados a três condições: fatores gestacionais e maternos; condições de nascimento e prematuridade; e fatores relacionados ao ambiente da UTIN.

\section{Fatores Gestacionais e Maternos}

A relação entre a presença de fatores de risco maternos e a possibilidade de infecção no recémnascido é evidente em diversos estudos desta revisão, com ênfase à infecção no período gestacional e a ruptura prematura de membranas amnióticas.

Um estudo realizado no estado do Amazonas identificou que a colonização pelo microorganismo Streptococcus do grupo B (GBS) nas gestantes aumenta em 15,2\% o risco de sepse neonatal em prematuros $^{(4)}$. Outros achados de delineamento retrospectivo referem associação entre infecção materna por GBS no trato urinário $(62,1 \%)$ com prevalência de sepse neonatal em 1/1.000 nascidos vivos na unidade de terapia intensiva, e ainda que outras infecções originadas durante a gestação sejam fatores de risco para a sepse neonatal ${ }^{(5)}$.

Corroborando com os achados dessa investigação, estudos relatam que a frequência de sepse neonatal após ruptura de membranas superior a 18 horas é de aproximadamente $72,7 \%$, com risco aumentado quando associada a si $\neg$ nais e sintomas de corioamnionite ${ }^{(6)}$. Estima-se que a rup $\neg$ tura prematura de membranas amnióticas ocorra em aproximadamente $20 \%$ a $25 \%$ das gestações, sendo mais comum em mulheres colonizadas por $\mathrm{GBS}^{(5-7)}$, e evidencia-se como um importante fator de risco para prematuridade e sepse precoce, bem como principal causas de mortalidade e morbidade entre os recém-nascidos em todo o mundo ${ }^{(7-8)}$.

Por fim, destaca-se a correlação entre o uso empírico de antibióticos em mulheres com ruptura prolongada de membranas com a diminuição na incidência de sepse neonatal ${ }^{(9)}$. Embora o risco de septicemia neonatal seja reduzido por esta conduta, ele ainda permanece de 5 a $8 \%$. No entanto, a decisão de iniciar antibiótico empírico em recém-nascidos deve ser baseada na presença ou ausência destes fatores maternos e neonatais ${ }^{(6,10)}$.

\section{Condições de Nascimento e Prematuridade}

A prematuridade e o baixo peso ao nascer constituem um dos principais fatores de risco para sepse neonatal ${ }^{(11-12)}$. Em consonância com os esses achados, um estudo realizado no Brasil identificou uma maior taxa de sepse neonatal precoce nos recém-nas $\neg$ cidos com idade gestacional entre 29 e 34 semanas e peso $\leq 1000 \mathrm{~g}$. Esses resultados reforçam que a prematuridade e o baixo peso ao nascer constituem um fator importante para a sepse ${ }^{(13)}$. Ademais, uma investigação realizada na Ásia identificou que há associação entre o diagnóstico da sepse neonatal com a presença da hemocultura positiva, baixo peso ao nascer e prematuridade ${ }^{(14-15)}$. Assim, é possível inferir que a prematuridade associada ao baixo peso ao nascer constituem fatores de risco importantíssimos para o desenvolvimento da sepse ${ }^{(12,16-17)}$.

Outros estudos revelam que o aumento da incidência para esta patologia se dá em neonatos filhos de mães adolescentes, que tiveram parto prematuro, e acompanhamento pré-natal inferior ao recomendado pelo Ministério da Saúde (seis consultas), fato que contribui para a ausência de dados da gestação considerados importantes na investigação desse processo infeccioso ${ }^{(4)}$.

\section{Fatores relacionados ao ambiente da UTIN}

A sepse tardia está relacionada com a permanência do recém-nascido na UTIN exposto aos riscos inerentes desse processo, tais como o uso do cateter central de inserção periférica (PICC), ventilação mecânica, e uso de nutrição parenteral ${ }^{(17,18-19)}$. Um estudo que analisou recém-nascidos que evoluíram para sepse por Klebsiella pneumoniae (ESBL) identificou que todos os participantes utilizaram cateter 
venoso central, nutrição parenteral prolongada e antibiótico de largo espectro; a maioria necessitou de ventilação mecânica e teve tempo de internação superior a 21 dias na unidade. Tais achados indicam que esses fatores contribuem para o aumento do risco de infecções na UTIN pela exposição aos diversos procedimentos invasivos, muitas vezes necessários para a terapia de alto risco. Esses procedimentos associados a manipulação constante dos neonatos e sua condição clínica eleva também o risco de mortalidade dessa população ${ }^{(20-21)}$.

Por fim, o uso da incubadora, nutrição intravenosa e uso do PICC também foram identificados como fatores de risco para a sepse neonatal, sendo o PICC o fator de maior gravidade ${ }^{(22-23)}$.

\section{CONCLUSÃO}

A análise aprofundada dos estudos permitiu a formalização dos fatores de risco que interferem no acometimento da sepse neonatal. A prematuridade e o baixo peso ao nascer prevaleceram em todos os estudos como fator preditivo de maior evidência. A idade gestacional, infecção do trato geniturinário materno e ruptura prematura das membranas amnióticas foram os principais fatores de risco gestacionais intervenientes na sepse neonatal. Os fatores relacionados ao ambiente da UTIN contemplam o tempo médio de permanência hospitalar, uso de dispositivos invasivos, tais como o cateter PICC, ventilação mecânica invasiva e nutrição parenteral, que são agravados pela imaturidade imunológica do recém-nascido.

O estudo apresenta limitações, uma vez que não foi evidenciada uma padronização dos métodos de investigação dos fatores associados à sepse neonatal. Dessa forma, os resultados das pesquisas podem variar de acordo com as características da população que foi investigada.

Portanto, conclui-se que a identificação dos fatores de riscos associados ao diagnóstico de sepse neonatal poderá subsidiar a realização de intervenções e demais pesquisas que colaborem para a diminuição da mortalidade neonatal gerada a partir desses riscos.

\section{REFERÊNCIAS}

1. Berardi A, Cattelani C, Creti R, Berner R, Pietrangiolillo Z, Immaculada M, et al. Group B streptococcal infections in the newborn infant and the potential value of maternal vaccination. Expert Rev Anti Infect Ther. [Internet] 2015; 13(11) [acesso em 15 jul 2015]. Disponível: http://dx.doi.org/10.1586/14787210.2015.1079126.

2. Ministério da Saúde (BR). Agência Nacional de Vigilância Sanitária. Neonatologia: critérios nacionais de infecção relacionada à assistência à saúde. Brasília (DF): Ministério da Saúde; 2010.

3. Verstraete EH, De Coen K, Vogelaers D, Blot S. Risk Factors for Health Care-Associated Sepsis in Critically III Neonates Stratified by Birth-Weight. Pediatr Infect Dis J. [Internet] 2015; 34(11) [acesso em 15 jul 2015] Disponível: http://dx.doi.org/10.1097/INF.0000000000000851.

4. Pinheiro RS, Ferreira LCL, Brum IR, Guilherme JP, Monte RL. Estudo dos fatores de risco maternos associados à sepse neonatal precoce em hospital terciário da Amazônia brasileira. Rev. Bras. Ginecol. Obstet. [Internet] 2007; 29(8) [acesso em 15 jul 2015]. Disponível: http://dx.doi.org/10.1590/S0100-72032007000800002.

5. Goulart AP, Valle CF, Dal-Pizzol F, Cancelier ACL. Fatores de risco para o desenvolvimento de sepse neonatal precoce em hospital da rede pública do Brasil. Rev. bras. ter. intensiva. [Internet] 2006; 18(2) [acesso em 15 jul 2015]. Disponível: http://dx.doi.org/10.1590/S0103-507X2006000200008.

6. Puopolo KM, Draper D, Wi S, Newman TB, Zupancic J, Lieberman E, et al. Estimating the Probability of Neonatal Early-Onset Infection on the Basis of Maternal Risk Factors. Pediatrics. [Internet] 2011; 128(5) [acesso em 15 jul 2015]. Disponível: http://pediatrics.aappublications.org/content/pediatrics/128/5/e1155.full.pdf.

7. Leal YA, Álvarez-Nemegyei J, Velázquez JR, Rosado-Quiab U, Diego-Rodríguez N, Paz-Baeza E, et al. Risk factors and prognosis for neonatal sepsis in southeastern Mexico: analysis of a four-year historic cohort followup. BMC Pregnancy Childbirth. [Internet] 2012; 12(48) [acesso em 15 jul 2015]. Disponível:

http://dx.doi.org/10.1186/1471-2393-12-48. 
8. Alam MM, Saleem AF, Shaikh AS, Munir O, Qadir M. Neonatal sepsis following prolonged rupture of membranes in a tertiary care hospital in Karachi, Pakistan. J Infect Dev Ctries. [Internet] 2014; 8(1) [acesso em 15 jul 2015 ]. Disponível: http://dx.doi.org/10.3855/jidc.3136.

9. Mukhopadhyay S, Puopolo KM. Risk assessment in neonatal early onset sepsis. Semin Perinatol. [Internet] 2012; 36(6) [acesso em 15 jul 2015]. Disponível: http://dx.doi.org/10.1053/j.semperi.2012.06.002.

10. Turrentine MA, Greisinger AJ, Brown KS, Wehmanen OA, Mouzoon ME. Duration of intrapartum antibiotics for group B streptococcus on the diagnosis of clinical neonatal sepsis. Infect Dis Obstet Gynecol. [Internet]. 2013; (2013) [acesso em 15 jul 2015]. Disponível: http://dx.doi.org/10.1155/2013/525878.

11. Yilmaz NO, Agus N, Helvaci M, Kose S, Ozer E, Sahbudak Z. Change in Pathogens Causing Late-onset Sepsis in Neonatal Intensive Care Unit in Izmir, Turkey. Iran J Pediatr. [Internet] 2010; 20(4) [acesso em 15 jul 2015$].$ Disponível: http://www.ncbi.nlm.nih.gov/pmc/articles/PMC3446087/pdf/IJPD-20-451.pdf.

12. Chan GJ, Lee ACC, Baqui AH, Tan J, Black RE. Risk of early-onset neonatal infection with maternal infection or colonization: a global systematic review and meta-analysis. PloS Med. [Internet] 2013; 10(8) [acesso 15 jul 2015]. Disponível: http://dx.doi.org/10.1371/journal.pmed.1001502.

13. Carvalho JK, Moore DB, Luz RA, Xavier-Elsas PP, Gaspar-Elsas MIC. Prediction of sepsis-related outcomes in neonates through systematic genotyping of polymorphisms in genes for innate immunity and inflammation: a narrative review and critical perspective. São Paulo Med. J. [Internet] 2013; 131(5) [acesso em 15 jul 2015]. Disponível: http://dx.doi.org/10.1590/1516-3180.2013.1315519.

14. Karambin M, Zarkesh M. Entrobacter, the most common pathogen of neonatal septicemia in rasht, Iran. Iran J Pediatr. [Internet] 2011; 21(1) [acesso em 15 jul 2015]. Disponível:

http://www.ncbi.nlm.nih.gov/pmc/articles/PMC3446115/pdf/IJPD-21-083.pdf.

15. Seale AC, Mwaniki M, Newton CR, Berkley JA. Maternal and early onset neonatal bacterial sepsis: burden and strategies for prevention in sub-Saharan Africa. Lancet Infect Dis. [Internet] 2009; 9(7) [acesso em 15 jul 2015]. Disponível: http://dx.doi.org/10.1016/S1473-3099(09)70172-0.

16. Gessner BD, Castrodale L, Soriano-Gabarro M. Aetiologies and risk factors for neonatal sepsis and pneumonia mortality among Alaskan infants. Epidemiol Infect. [Internet] 2005; 133(5) [acesso em 15 jul 2015]. Disponível: http://dx.doi.org/10.1017/S0950268805004449.

17. Meireles LA, Vieira AA, Costa CR. Avaliação do diagnóstico da sepse neonatal: uso de parâmetros laboratoriais e clínicos como fatores diagnósticos. Rev. esc. enferm. USP. [Internet] 2011; 45(1) [acesso em 15 jul 2015 ]. Disponível: http://dx.doi.org/10.1590/S0080-62342011000100005.

18. Romanelli RMC, Anchieta LM, Carvalho EAA, Glória e Silva LF, Nunes RVP, Mourão PH, et al. Risk factors for laboratory-confirmed bloodstream infection in neonates undergoing surgical procedures. Braz. J. Infect. Dis. [Internet] 2014; 18(4) [acesso em 15 jul 2015]. Disponível: http://dx.doi.org/10.1016/j.bjid.2013.12.003.

19. Marchant EA, Boyce GK, Sadarangani M, Lavoie PM. Neonatal sepsis due to coagulase-negative Staphylococci. Clin Dev Immunol. [Internet] 2013;(2013) [acesso em 15 jul 2015]. Disponível: http://dx.doi.org/10.1155/2013/586076.

20. Tragante CR, Ceccon MEJR, Falcão MC, Seiti M, Sakita N, Vieira RA. Prevalência de sepse por bactérias Gram negativas produtoras de beta-lactamase de espectro estendido em Unidade de Cuidados Intensivos Neonatal. Rev. paul. pediatr. [Internet] 2008; 26(1) [acesso em 15 jul 2015]. Disponível:

http://dx.doi.org/10.1590/S0103-05822008000100010.

21. Hsieh E, Smith PB, Jacqz-Aigrain A, Kaguelidou F, Cohen-Wolkowiez M, Manzoni P, et al. Neonatal fungal infections: when to treat?. Early Hum Dev. [Internet] 2012; 88(Suppl 2) [acesso em 15 jul 2015]. Disponível: http://dx.doi.org/10.1016/S0378-3782(12)70004-X.

22. Li Z, Xiao Z, Li Z, Zhong Q, Zhang Y, Xu F. 116 cases of neonatal early-onset or late-onset sepsis: A single center retrospective analysis on pathogenic bacteria species distribution and antimicrobial susceptibility. Int J Clin Exp Med. [Internet] 2013; 6(8) [acesso 15 jul 2015]. Disponível:

http://www.ncbi.nlm.nih.gov/pmc/articles/PMC3762626/.

23. Lin CB, Hornik CP, Clark R, Cotten CM, Benjamin Jr DK, Cohen-Wolkoweiz M, et al. Very low birth weight 
neonates who survive early-onset sepsis do not have an increased risk of developing late-onset sepsis. Early Hum Dev. [Internet] 2012; 88(11) [acesso em 15 jul 2015]. Disponível: http://dx.doi.org/10.1016/j.earlhumdev.2012.07.009.

24. Jiang Z, Ye GY. 1:4 matched case-control study on influential factor of early onset neonatal sepsis. Eur Rev Med Pharmacol Sci. [Internet] 2013; 17(18) [acesso em 15 jul 2015]. Disponível:

http://www.europeanreview.org/article/5191.

25. Borges RM, Soares LR, de Brito CS, Brito DVD, Abdallah VOS, Gontijo Filho PP. Fatores de risco associados à colonização por Candida spp em neonatos internados em uma Unidade de Terapia Intensiva Neonatal brasileira. Rev. Soc. Bras. Med. Trop. [Internet] 2009; 42(4) [acesso em 15 jul 2015]. Disponível:

http://dx.doi.org/10.1590/S0037-86822009000400014.

26. Madan JC, Salari RC, Saxena D, Davidson L, O'Toole GA, Moore JH, et al. Gut microbial colonisation in premature neonates predicts neonatal sepsis. Arch Dis Child Fetal Neonatal Ed. [Internet] 2012; 97(6) [acesso 15 jul 2015]. Disponível: http://dx.doi.org/10.1136/fetalneonatal-2011-301373. 